\title{
Basalt fibers as functional additives in coating of textiles
}

\author{
Carolin Ruffen, Boris Mahltig $\mathbb{1}$
}

(C) The Author(s) 2020

\begin{abstract}
In the presented study, basalt fibers are used as additives in polymer coatings applied onto textiles substrates. As basalt fibers, short fibers with diameters of around $13 \mu \mathrm{m}$ and with lengths in the range of $0.16-4 \mathrm{~mm}$ are used. As the textile substrate, a cotton fabric is used. The polymer coatings are based on acrylate or polyurethane. As a further coating additive, the silicate compound Aerosil is also evaluated. The realized coated materials are investigated by scanning electron microscopy (SEM) and by optical spectroscopy in arrangement of diffusive reflection and transmission. The optical spectra are recorded in a spectral range from 220 to $1400 \mathrm{~nm}$. By these optical measurements, it is demonstrated that the optical properties for UV-light, visible light and near infrared light are influenced by the basalt-containing coatings. The transmission for UV-light is drastically reduced. It is possible to decrease the transmission for infrared light drastically by use of these coating while the IRreflectance stays on a significant level. In future perspective, these types of coatings could be used as protection against both UV- and IR-light. The surface topography of realized coatings is dominated by the applied basalt fibers. In the case of medium or high basalt fiber concentration, a parallel orientation of some basalt fibers occurs. This type of parallel arrangement could be used in future perspective for different types of applications, e.g., the development of new filter materials.
\end{abstract}

Keywords Basalt fibers, Inorganic fibers, Textile coating, Cotton, UV protective, Infrared protection

C. Ruffen, B. Mahltig ( $\square$ )

Faculty of Textile and Clothing Technology, Niederrhein

University of Applied Sciences, Webschulstr. 31, 41065

Mönchengladbach, Germany

e-mail: Boris.mahltig@hs-niederrhein.de

\section{Introduction}

Basalt fibers are inorganic fibers that are originally produced by using stones from volcanic rocks. These stones are molten and during a spinning process, are transferred to fiber materials. In a certain way, basalt fibers are similar to glass fibers. ${ }^{1-4}$ However, there are some main differences, which are related to the origin from natural stones. ${ }^{5,6}$ In contrast to glass fibers, the basalt fibers contain the main components silica and alumina and also significant amounts of the iron oxides $\mathrm{FeO}$ and $\mathrm{Fe}_{2} \mathrm{O}_{3}$. The content of iron oxide in basalt fibers differs depending on the origin of the basalt stones used for the fiber production. There are different values for the iron oxide content in basalt fibers reported in the range of $6-16 \mathrm{wt} \% .^{3,4,7-9}$ Due to this iron oxide content, the basalt fibers exhibit a brown coloration. Also, a stronger absorption of heat radiation is reported. ${ }^{4,9}$ Beside iron oxide, basalt fibers also contain around $1 \%$ titanium dioxide. ${ }^{4,6,7}$

There are manifold applications reported for basalt fibers. One main application is the use as a reinforcing fiber as a part of composite materials. ${ }^{9}$ Basalt fibers are used to reinforce thermoplastic polymers such as polypropylene. ${ }^{10,11}$ Also, resins based on reactive components like epoxy-containing compounds are reported to be reinforced by basalt fibers. ${ }^{12}$ Beside the reinforcing of organic polymers, the basalt fibers are also used as a strengthening material for concrete structures. ${ }^{13,14}$ These reinforced concrete materials can be named as an inorganic/inorganic composite material. Basalt fibers are used as well for preparation of ropes and rods which are applied in building engineering. ${ }^{15}$ Also, applications of basalt fibers related to traditional clothing are possible. ${ }^{16}$ A good example in this field is basalt fiber fabrics equipped with aluminum foils for the use as heat protective clothing. ${ }^{17-19}$ 
Basalt fiber materials are used for applications in heat protection and protection against infrared and UV-radiation. ${ }^{20,21}$ The protection against laser beams is evaluated and reported in the literature. ${ }^{22}$ All these reported studies are related to woven and nonwoven materials, multilayer materials or composites. Short basalt fibers are reported for their use as an additive in epoxy-based coating on steel substrates to improve to fire protection performance of the coated steel. ${ }^{23}$

With this background, one main motivation of the current study is to evaluate basalt fibers as simple coating additives for coating on textiles, with the question, if these coatings can also lead to UV- and infrared protective properties.

The use of basalt and glass fibers as coating additives is reported for epoxy coatings placed on metal substances. ${ }^{23,24}$ In these applications, the main aim is the protection of the coated metal substrate by the coating. This protection is investigated in relation to temperature and chemical exposure. In contrast to the actual performed investigation, no application on textile substrates was done. Also, no measurements with optical spectroscopy are reported.

A well-established technique using short fibers in coatings on textiles is the flock technology. ${ }^{25,26}$ In this technique, mostly short fibers of polyamide but also viscose fibers are placed in the presence of an electric field on a binder layer that was previously applied on a textile substrate. In this case, the orientation of the fibers on the substrates is determined by parameters such as the electrical field and the fiber length and diameter. The final optical properties are determined by the dyeing of the polyamide fibers. To the best of our knowledge, the application of basalt fibers in a flock coating on textile substrates is not reported up to now.

In contrast to most applications mentioned in the literature and other previously reported investigations on basalt fibers, the now performed investigation is related to a new type of application for basalt fibers. The topic of the actual study is the use of short basalt fibers as additives in coatings applied onto textile substrates.

Short basalt fibers with lengths from 0.16 to $4 \mathrm{~mm}$ are evaluated as an additive to a coating recipe for application onto cotton fabrics. In a first approach, these basalt fibers can be understood as a special kind of inorganic component or pigment which is part of the coating. Analogous to effect pigments, the short basalt fibers are anisotropic materials containing a length which is 100-1000 times longer compared to the diameter of the basalt fibers. ${ }^{27}$ As reported for other inorganic pigments, by use of basalt short fibers, the interaction of the coated textile to UV light, visible light and infrared light can be manipulated. ${ }^{27-29}$ For this reason, the optical properties of the realized basalt modified coatings are intensively investigated.

A decrease in transmission for UV light and infrared light can be used for the development of protective material against UV- and IR-light. For this, applica- tions in the field of radiation protection and heat protection could be aimed by the developed coated materials. Due to the natural brown coloration of the basalt fibers, applications in the field of visible and near infrared camouflage textiles could also be developed on the base of currently presented coatings. ${ }^{30}$ Also, the surface topography of the prepared coatings is investigated by scanning electron microscopy (SEM). The surface topography of basalt fiber coatings shows for some samples a parallel orientation of the deposited basalt fibers. The degree of orientation depends on the amount of applied basalt fibers. This parallel orientation of some basalt fibers to each other is expected as a result of processes during the coating and drying procedure.

\section{Experimental section}

\section{Materials}

As basalt fibers, UCF fibers (uncoated chopped fibers) are used. These basalt fibers are supplied by the company Deutsche Basalt Faser GmbH (Sangerhausen, Germany). According to supplier information, the diameter of all used basalt fiber types is $13 \mu \mathrm{m}$. Altogether, three different types of basalt fibers are investigated, which have different fiber lengths of 0.16 , 1.6 or $4 \mathrm{~mm}$, according to supplier information. A photographic image of the used basalt fibers with a length of $4 \mathrm{~mm}$ is shown in Fig. 1. The typical brown coloration of basalt fibers is obvious. SEM images of the used basalt fibers of all three lengths are presented in Fig. 2 in two different magnifications. By view of these SEM images, it is clear that the basalt fibers containing $1.6 \mathrm{~mm}$ length are roughly ten times longer compared to fibers with length of $0.16 \mathrm{~mm}$. However, for all types of fibers, fiber pieces with significantly shorter length or broken pieces are observed. These

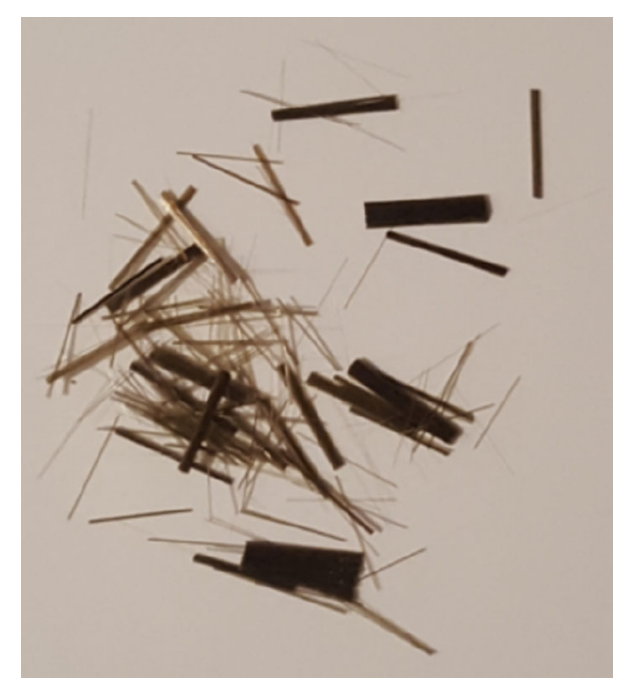

Fig. 1: Photograph of used basalt fibers, length $4 \mathrm{~mm}$ 


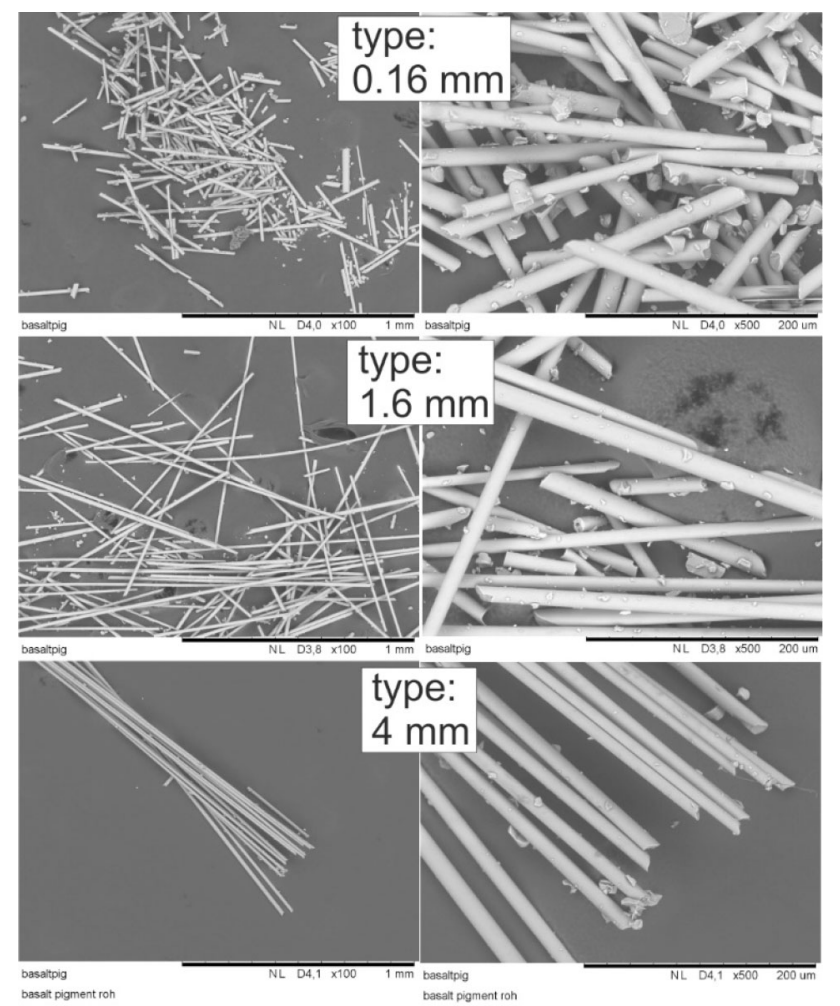

Fig. 2: SEM images of used basalt fibers. Compared are the basalt fiber types with different lengths. For each fiber type, images of two different magnifications are shown

smaller fibers or broken pieces are probably the result of mechanical influences appearing after fiber production. As a result of the mechanical forces, e.g., occurring during storage, package or transportation, some fibers break into smaller pieces. Beside the length of the basalt fibers, the fiber diameter also varies in a certain range around the value of $13 \mu \mathrm{m}$, which is given by the supplier. According to supplier information, these basalt fibers are dedicated for the use as additive in polymers to improve thermal and mechanical properties of modified polymers. For current investigations, two different types of binders are used for coating preparation on the textile fabric. First, the acrylate-based binder Printperfekt $226 \mathrm{FF}$ is used. This binder is supplied by the CHT R.Beitlich $\mathrm{GmbH}$ (Tübingen, Germany). Second, the polyurethane binder Printperfekt Base FF is used, which is supplied by the same company. For some preparations, the application with the binder Printperfekt 226FF is modified with the silicate compound Aerosil COK 84 (Evonik, Germany). As the textile substrate, a white plain weave cotton fabric is used.

\section{Preparation}

For coating preparation, at first a basalt fiber-containing printing paste is prepared. For this, the basalt fibers are stirred into the used binders in different concentrations in the range from 2.5 to $50 \mathrm{wt} \%$. If the recipe is modified by adding the silicate compound Aerosil COK 84, the Aerosil is added at first to the basalt fibers in a concentration of $2 \mathrm{wt} \%$. The application of the printing paste onto the textile substrate is done by using a Mathis Labdryer type LTE which is equipped with a coating device (supplied by the company Werner Mathis AG, Switzerland). The coating application is done in two layers. After application of each layer, the samples are dried at $80^{\circ} \mathrm{C}$ for a duration of $1 \mathrm{~min}$.

\section{Analytics}

The topography of coated samples is investigated using a scanning electron microscope (SEM) Tabletop TM3000 supplied by Hitachi (Japan). SEM images of sample cross sections are recorded by an SEM TM4000plus Tabletop Microscope (Hitachi, Japan). Optical spectra are recorded from the samples in arrangement of diffusive reflection and diffusive transmission. For these optical measurements, a photo spectrometer UV-2600 from Shimadzu (Japan) is used. This spectrometer is equipped with an integrated sphere enabling the measurements in diffusive arrangement. The optical measurements are done in a spectral range from 220 to $1400 \mathrm{~nm}$. For documentation of color properties of prepared samples, the CIE $L^{*} a^{*} b^{*}$ indices are measured. These measurements are done with a device DATA Color 400 (Rotkreuz, Switzerland).

\section{Results and discussions}

\section{Microscopic investigations}

The microscopic measurements of the prepared coatings are done by scanning electron microscopy (SEM) to investigate the surface topography of realized coated fabrics. Beside the surface topography, the used SEM device also enables the depiction of a material contrast, so the deposited basalt fibers are shown in brighter contrast compared to the polymeric binder and the textile substrate. The material contrast is related to the inorganic content of the basalt fibers containing mainly silica and alumina. Silicon $\mathrm{Si}$ and aluminum $\mathrm{Al}$ are chemical elements of higher weight, which leads to a different material contrast compared to the polymer binders and the cotton fabric containing mainly the chemical elements carbon $\mathrm{C}$, oxygen $\mathrm{O}$ and hydrogen $\mathrm{H}^{31}$

The SEM images of cotton fabrics carrying the acrylate coating with the basalt fibers are shown in Fig. 3. In Fig. 3, samples with an increasing content of basalt fibers from 2.5 up to $50 \%$ in the coating recipe are compared. These samples are prepared by using 

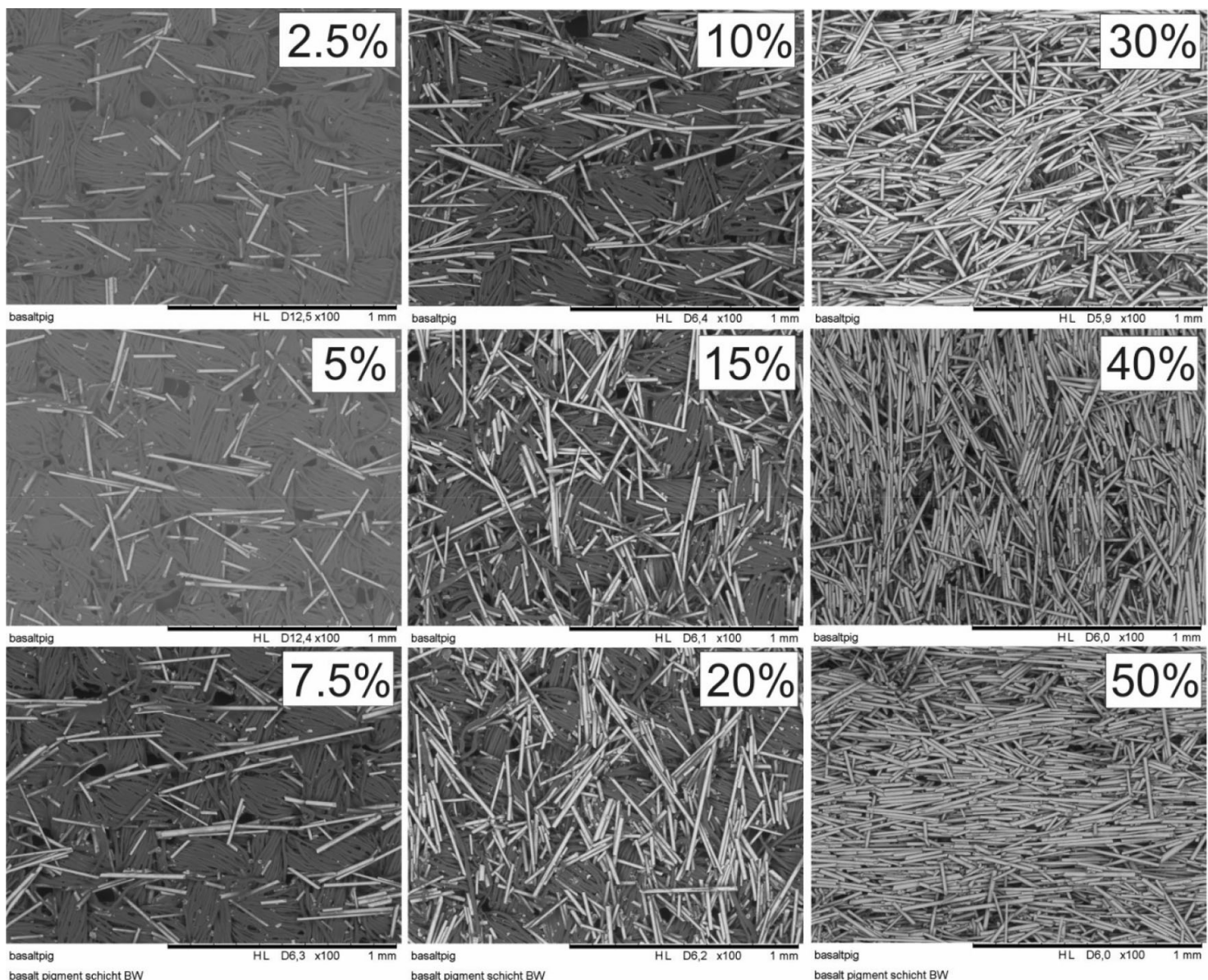

Fig. 3: SEM images of basalt fiber-containing acrylate coatings on cotton fabric. The basalt fibers contain $0.16 \mathrm{~mm}$ length and are added in increasing concentrations to the coating recipe. The basalt fiber content is indicated in each image

fibers of shortest length $0.16 \mathrm{~mm}$. With these short fibers, stable coating recipes can be prepared even if the fiber concentration is set to high values of $50 \%$. If the basalt fiber concentration is $30 \%$ or higher, the complete fabric is covered by the fibers. In the case of the lowest investigated concentrations of $2.5 \%$ and $5 \%$, the basalt fibers are nearly randomly oriented on the cotton substrate. With use of higher fiber concentration in the coating recipe, a trend to a parallel orientation of many basalt fibers can be observed (Fig. 3).

The occurring parallel orientation could be the result of an arrangement occurring during application of the coating recipe and its drying on the textile substrate. It could be assumed that the deposited basalt fibers have a certain influence on the orientation of the next deposited basalt fibers. If the basalt fibers are applied in low concentration, the distance between the single deposited basalt fibers is large, so contact between the deposited basalt fibers occurs in smaller amounts and the fibers are more randomly distributed. By applying a medium or high concentration of basalt fibers, the distance between the fiber in the coating is decreased and a parallel orientation of some basalt fibers occurs.
A cross section of a coated fabric is presented in Fig. 4. This SEM image shows a cross section of a cotton fabric with a basalt fiber-containing acrylate coating. The basalt fibers are $0.16 \mathrm{~mm}$ in length and are added in a concentration of $10 \%$ to the coating recipe. All three types of materials are clearly visible in the microscopic images - the basalt fibers, the fibers of the cotton fabric and the binder in between both fiber types. The thickness of the coated cotton substrate is around $200 \mu \mathrm{m}$. The coating on top of this substrate is around $25 \mu \mathrm{m}$ thick. This top part of the coating contains the unbroken basalt fibers. The acrylic binder and smaller pieces of broken basalt fibers can penetrate deeper into the free areas of the cotton fabric, so for these areas altogether, thicknesses of up to $100 \mu \mathrm{m}$ can be reached (Fig. 4).

Acrylate coating recipes prepared with basalt fibers containing a longer fiber length of $1.6 \mathrm{~mm}$ or $4 \mathrm{~mm}$ are realized and applied with a basalt fiber concentration of $2.5 \%$. With higher concentrations, the stability of the coating recipe is limited due to occurring segregation of the coating recipe. The related SEM images of coated samples with longer basalt fibers are presented in Fig. 5. It can be determined that many of the longer 

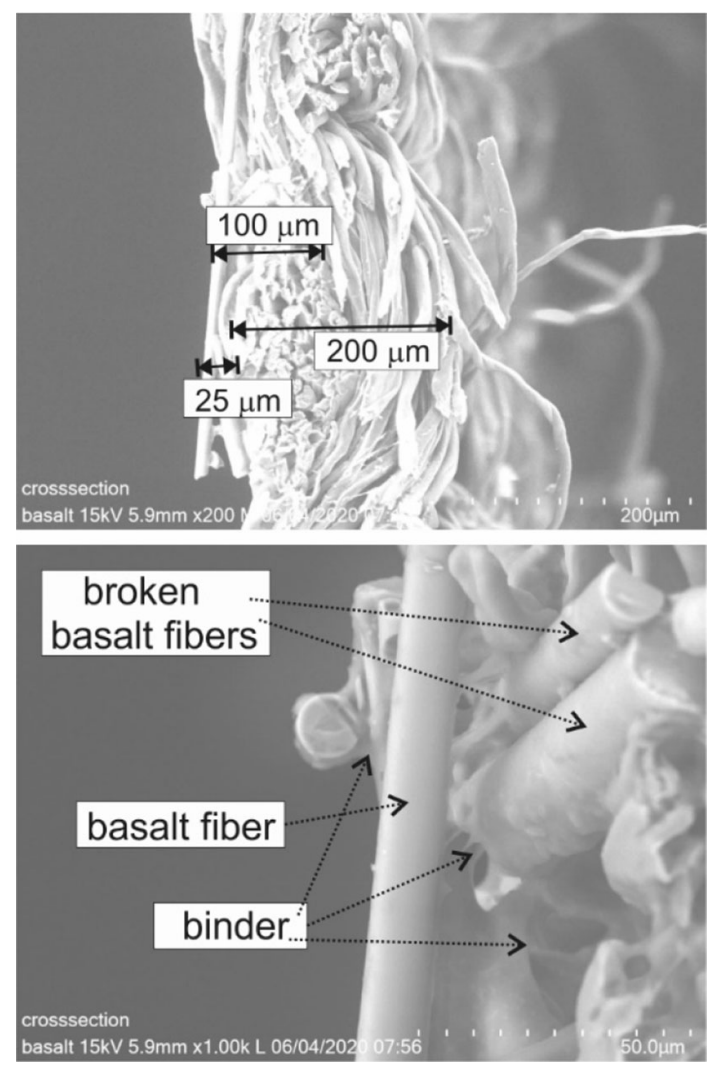

Fig. 4: SEM images of a cross section of a cotton fabric with basalt fiber-containing acrylate coating. The basalt fibers contain $0.16 \mathrm{~mm}$ length and are added in a concentration of $10 \%$ to the coating recipe. The image is shown in two different magnifications

fibers are broken into smaller pieces, likely due to mechanical influences during recipe preparation and application of the coating onto the textile surface. Also, the hand feeling of cotton substrates changed drastically after coating with longer basalt fibers. These coated cotton fabrics exhibit a hard and scratchy hand feeling making them unsuitable for any clothing application.

Additionally, to the simple acrylate binder, coating recipes based on a polyurethane binder and an acrylate binder modified with the silicate Aerosil are also evaluated. The related SEM images are depicted in Figs. 6 and 7. Figure 6 shows SEM images in low magnification of basalt fiber with $0.16 \mathrm{~mm}$ length added in a concentration of $20 \%$ to different binder systems. It is clearly seen that with different binder systems, a parallel orientation of some basalt fibers occurs. For this, it can be stated that the above-mentioned orientation of some basalt fibers during the coating process is independent from the type of used binder.

In Fig. 7, SEM images in higher magnification are presented. Also, in these higher magnified images, the parallel orientation of some basalt fibers is observed. In all images, smaller pieces of basalt material are

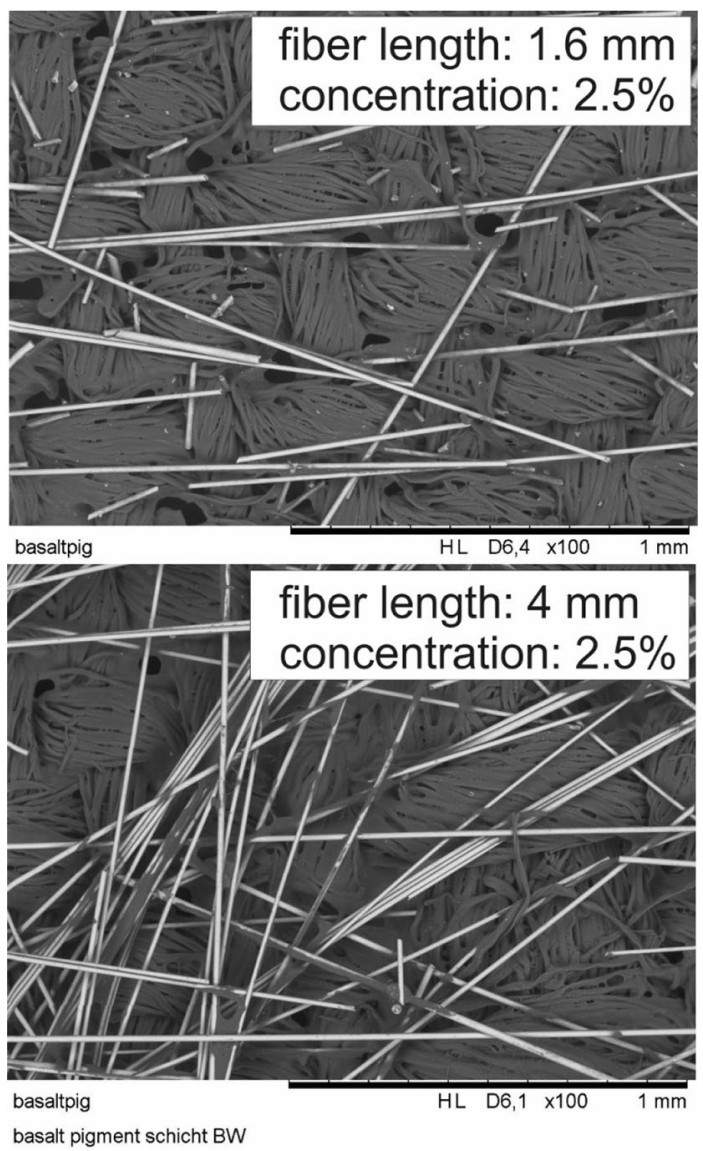

Fig. 5: SEM images of basalt fiber-containing acrylate coatings on cotton fabric. Compared are the basalt fiber types with different lengths. The concentration of basalt fibers in the coating recipe is set to $2.5 \%$

observed. These smaller basalt pieces are obviously residues from broken basalt fibers, which are destructed during the recipe preparation and the coating application. For the acrylate recipe containing the silicate additive Aerosil, the Aerosil particles can also be detected in the related SEM image (Fig. 7). The Aerosil particles are regularly distributed in the binder layer.

\section{Spectroscopic investigations}

The optical properties of realized coated samples are investigated by using optical spectroscopy with wavelengths of light in the range of $220-1400 \mathrm{~nm}$. The aim of this investigation is the evaluation of the samples related to their interaction with different types of light. First, the view to the investigated region of UV light from 220 to $400 \mathrm{~nm}$ is done to evaluate if the applied coatings can act as a UV-protective material. Second, the investigation related to the area of visible light from $400 \mathrm{~nm}$ to $750 \mathrm{~nm}$ is done to gain information according to the coloration of the prepared coatings. These coloration measurements are accomplished by 

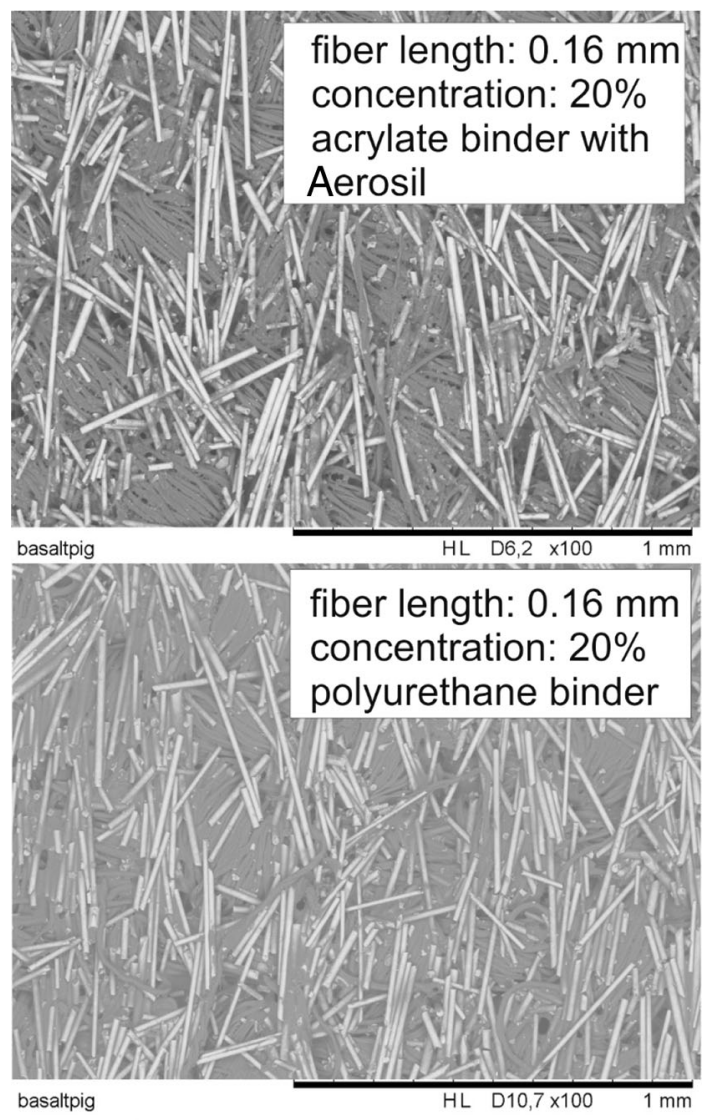

basalt pigment schicht BW

Fig. 6: SEM images basalt fiber-containing coatings using two different binder recipes on cotton fabric. The basalt fibers contain $0.16 \mathrm{~mm}$ length and are added with a concentration of $20 \%$ the coating recipe

determination of the CIE $L^{*} a^{*} b^{*}$ indices. Third, the view to the investigated region of IR light from 750 to $1400 \mathrm{~nm}$ is done to determine if the applied coatings can influence the interaction to IR light and can lead to an application as a heat protective material.

The performed spectroscopic investigations are done in two different arrangements-reflection or transmission-due to different purposes. The reflection measurements give mainly information on the color change after coating application, while the transmission is related to radiation protective properties. For reference measurements, an uncoated cotton fabric is used.

Determined reflection spectra of the coated fabrics are presented in Figs. 8 and 10. Figure 8 summarizes the reflection spectra of all samples which are acrylate based and contain basalt fibers with a length of $0.16 \mathrm{~mm}$. Here, the reflection spectra are compared due to the different content of basalt fiber concentration from $2.5 \%$ up to $50 \%$. It is obvious that the applied coatings decrease the reflection over the complete investigated spectral range from 220 to $1400 \mathrm{~nm}$. This decrease in reflection is clearly correlated to the amount of added basalt fibers to the
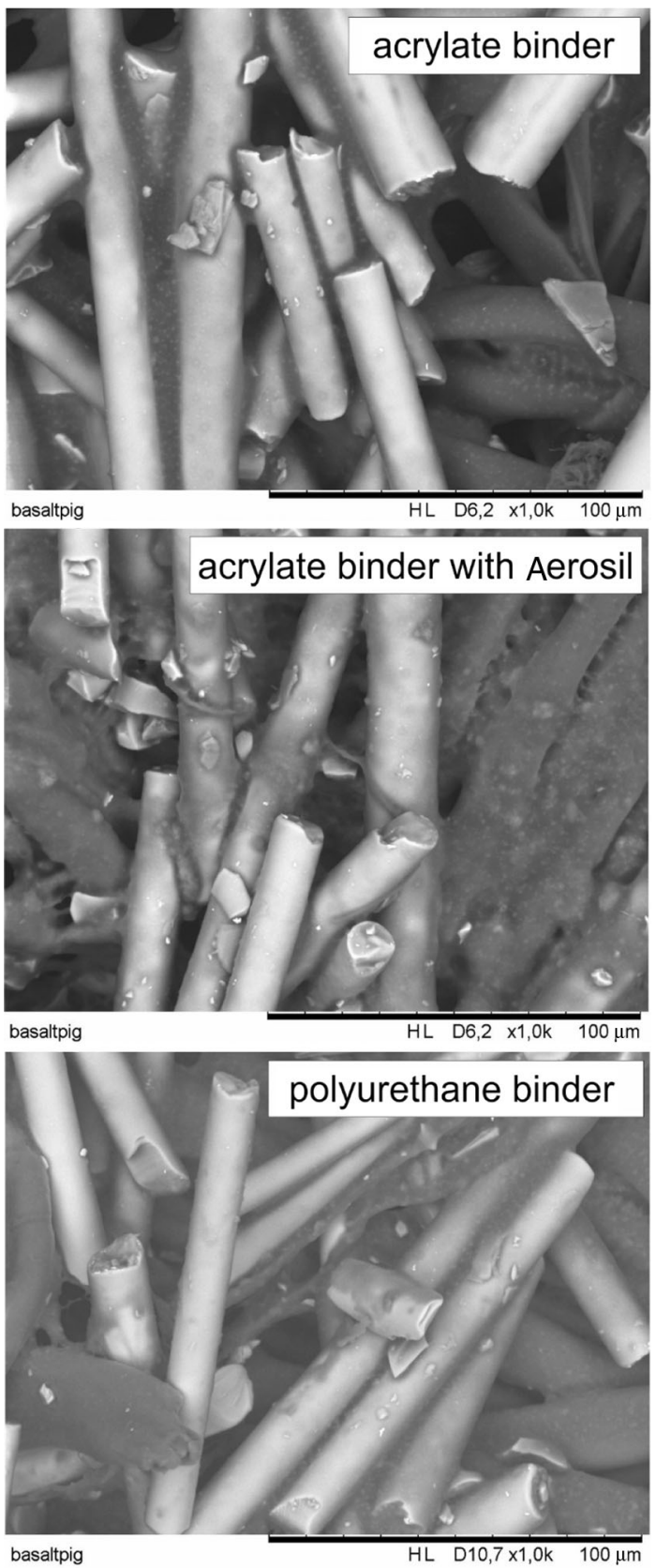

basalt pigment schicht BW

Fig. 7: SEM images with higher magnification of basalt fiber-containing coatings using three different binder recipes on cotton fabric. The basalt fibers contain $0.16 \mathrm{~mm}$ length and are added with a concentration of $20 \%$ the coating recipe

coatings. Because of the brown coloration of the basalt fibers themselves, the decrease in reflection in the visible range of light can be expected. Also, the higher reflection values for red light compared to lower reflection values for light in the range of $400-500 \mathrm{~nm}$ is related to the brown coloration of the basalt fibers. Applications of coatings with basalt fiber concentration of $30 \%$ or more lead to dark brown colored samples. 
Beside this influence on visible light, the reflection of UV-light is even more strongly decreased (Fig. 8). Samples with high basalt fiber content of $30 \%$ or more exhibit reflection values smaller than $5 \%$ over nearly the complete UV-range. Compared to the decrease in reflection for UV-light and visible light, the reflection of IR-light is less decreased by the applied coatings. For this, a stronger infrared reflection compared to the other types of investigated light can be stated.

Figure 9 shows a data evaluation of the reflection spectra presented in Fig. 8. In Fig. 9, the reflection

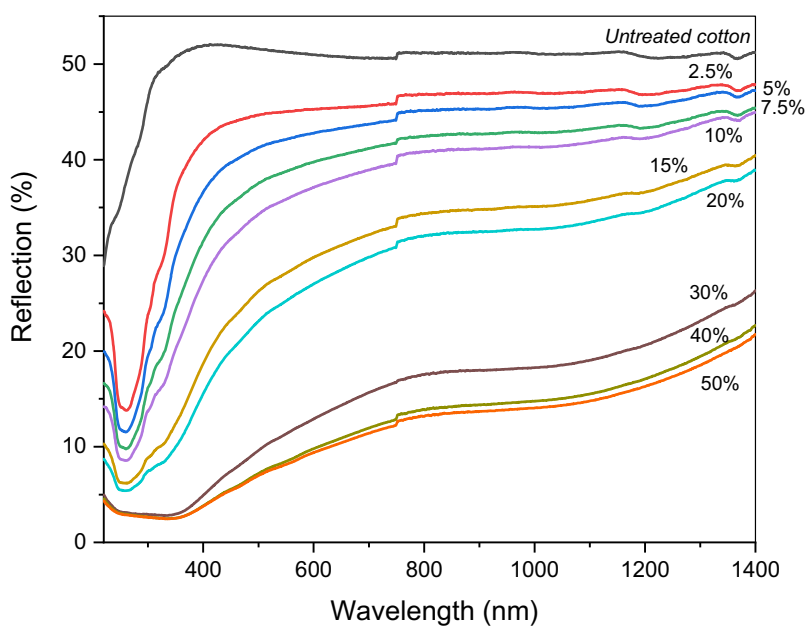

Fig. 8: Optical spectra of diffusive reflection. Compared are acrylate-based coatings containing basalt fibers with $0.16 \mathrm{~mm}$ length with increasing basalt fiber concentration in the coatings. The reflection spectrum of the untreated cotton fabric is shown as a reference

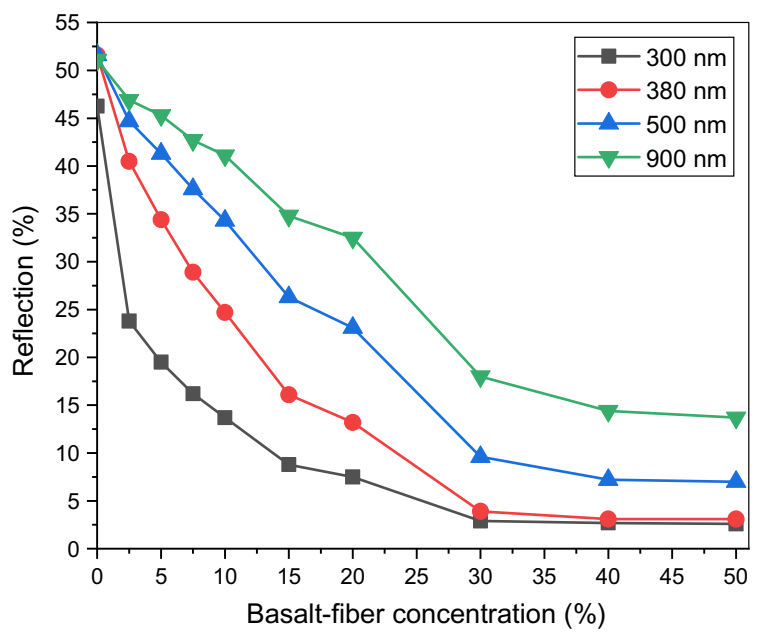

Fig. 9: Values of diffusive reflection taken at four different wavelengths of the optical spectrum as a function of the basalt fiber concentration of an applied coating. These coatings are acrylate based containing basalt fibers with $0.16 \mathrm{~mm}$ length with increasing basalt fiber concentration in the coatings values at four different wavelengths of the recorded spectra are shown as a function of the basalt fiber concentration in the applied coating. As four wavelengths, the values at 300 and $380 \mathrm{~nm}$ (both representative for UV-light), $500 \mathrm{~nm}$ for visible light and $900 \mathrm{~nm}$ for IR-light are selected. It is clearly seen that there is a strong decrease in reflection as a function of the basalt fiber concentration up to a concentration of $30 \%$. A further increase in the basalt concentration to values up to $50 \%$ does not lead to a further strong decrease in reflection values. For this, it can be stated, that for basalt concentrations in the range of $2.5-30 \%$, an increase in basalt fiber component leads to a progressed covering of the white cotton fabric by the brown colored basalt fibers. Here an increase in basalt concentration has a direct influence in the reflection properties. For concentrations of $30 \%$ or higher, the cotton fabric is nearly completely covered by the basalt fibers, so a further addition of more basalt component does not lead to a further change in coloration of the sample.

In Fig. 10, the reflection spectra of samples with the same concentration of basalt fibers $(20 \%)$ but with different binder recipes are compared. It is clearly seen that the type of used binder has nearly no influence on the optical properties of the realized coated samples. It can be stated that the optical active component in these coatings is the basalt fiber.

Determined transmission spectra of the coated fabrics are presented in Figs. 11 and 13. Figure 11 summarizes the transmission spectra of all samples which are acrylate based and contain basalt fibers with a length of $0.16 \mathrm{~mm}$. Here, the transmission spectra are compared due to the different basalt fiber concentrations from 2.5 up to $50 \%$. The transmission of UV light in the range from 220 to $300 \mathrm{~nm}$ is drastically reduced

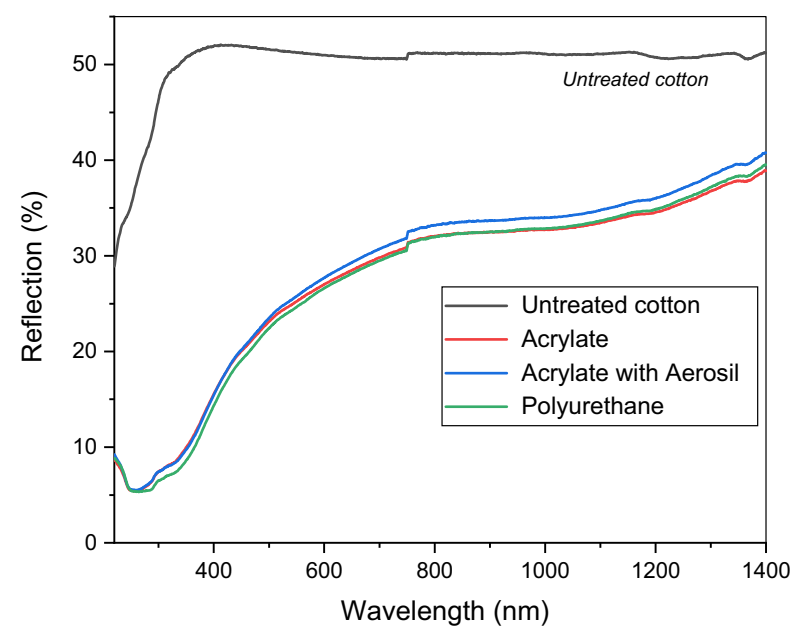

Fig. 10: Optical spectra of diffusive reflection. Compared are basalt fiber-containing coatings with different binder recipes. The used basalt fibers contain a length of $0.16 \mathrm{~mm}$ and are added to the coating recipe in a concentration of $20 \%$. The reflection spectrum of the untreated cotton fabric is shown as a reference 
even if only low amounts of basalt fibers are added to the coating. With high basalt fiber concentration of $30 \%$ or more, the transmission in this spectral range is near zero. For this, it can be assumed that the basalt fibers strongly absorb UV light with wavelength of $300 \mathrm{~nm}$ or shorter. Nevertheless, a different result is gained for the UV area between 300 and $400 \mathrm{~nm}$. This observed transmission behavior is in good accordance to the transmission reported for nonwoven fabrics made from basalt fibers. ${ }^{24}$ Of course, in this area, the transmission of UV light is also decreased by the basalt fiber-containing coatings but, especially in the spectral range of $370-400 \mathrm{~nm}$, a complete decrease to very low transmission is only reached for highest basalt fiber concentration. It should be kept in mind that this spectral region is especially important for UV protection against sun light because the UV content of sun light occurs especially in this region. ${ }^{32}$ For this, an excellent UV protection is only possible by application of coatings containing the high basalt fiber concentration.

UV-protective properties can be reached by application of UV-light absorbing components, which are called UV-absorbers. Often, two kinds of UV-absorbers are distinguished, which are inorganic UVabsorbers like $\mathrm{TiO}_{2}$ and organic UV-absorbers. ${ }^{33,34}$ The UV-protective properties of inorganic UV-absorbers such as $\mathrm{TiO}_{2}$ or $\mathrm{ZnO}$ are determined by the band-gap of this absorber, and below the wavelength related to the energy of the band-gap, the UV-light is completely absorbed. ${ }^{33,35}$ Organic UV-absorbers are comparable with organic dye stuffs and absorb UVlight of a certain spectral range. ${ }^{33}$ Beside $\mathrm{TiO}_{2}$ as an inorganic UV-absorber, the use of zinc oxide is also reported for the application of UV-protective properties on textiles. ${ }^{35-37}$

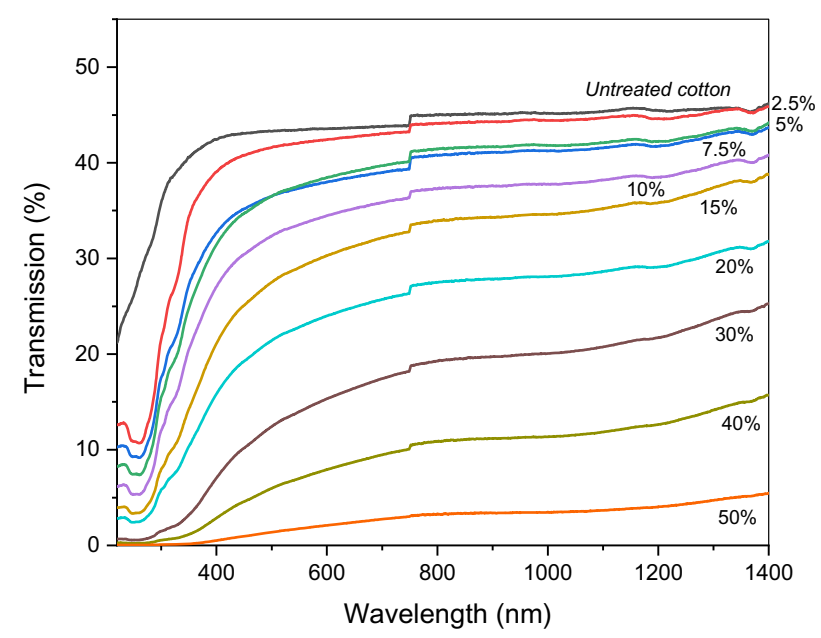

Fig. 11: Optical spectra of diffusive transmission. Compared are acrylate-based coatings containing basalt fibers with $0.16 \mathrm{~mm}$ length with increasing basalt fiber concentration in the coatings. The reflection spectrum of the untreated cotton fabric is shown as a reference
The basalt fiber-containing coating can be compared to applications using inorganic UV-absorbers. The basalt fibers are also an inorganic material absorbing UV-light below a certain wavelength and give a good UV-protection for this spectral range. This UV-absorbing property of basalt fibers is also related to a certain content of $\mathrm{TiO}_{2}$ in this type of fiber. Due to the additional content of iron oxide in the basalt fibers, UV-light with wavelengths higher than the bad-gap of $\mathrm{TiO}_{2}$ and also visible light are absorbed by the basalt fiber-containing coatings. For this, especially in the spectral range between 370 and $400 \mathrm{~nm}$, the UVprotective properties of basalt-containing coatings are better compared to systems using only zinc oxide or titanium oxide. However, by using organic UV-absorbers, better UV-protective properties in the spectral range from 370 to $400 \mathrm{~nm}$ are reached on polymer foils and textiles. ${ }^{33,38}$

The transmission for the visible and infrared light is also decreased as a function of basalt fiber concentration in the coating. However, in contrast to the reflection, the transmission for visible and IR-light decreased with a nearly linear behavior as a function of basalt fiber concentration (Fig. 12). The reflection is related to the coverage of the textile substrate. In contrast, the transmission is not only depending on the coverage. Also, the content of basalt fiber in deeper areas of the coating leads to an absorption of light during its transfer through the coating. By this, a decrease in transmission occurs. With basalt fiber coatings, it is possible to create textiles with low transmission values for visible and IR light of smaller than $5 \%$, while the reflection, especially for IR-light, is still higher with values of $>15 \%$ (Figs. 9 and 12).

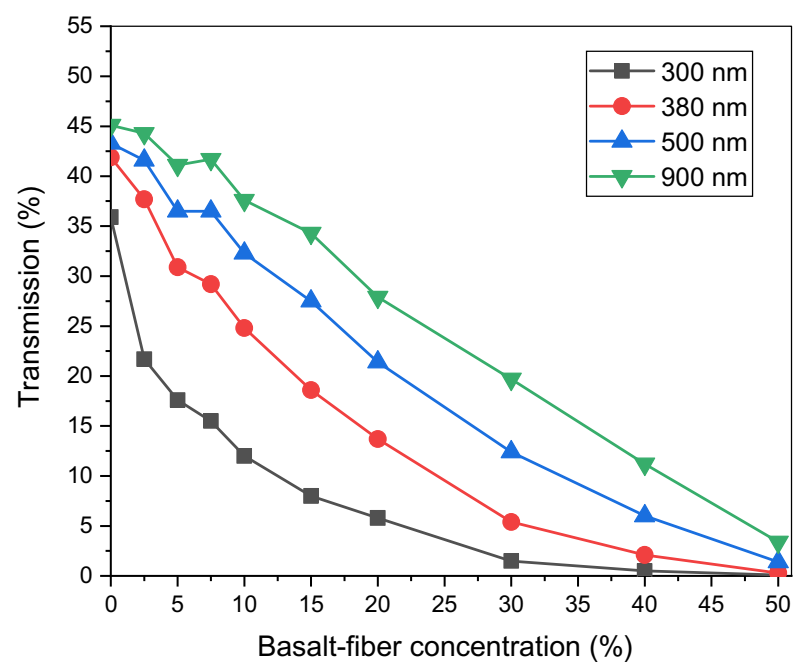

Fig. 12: Values of diffusive transmission taken at four different wavelengths of the optical spectrum as a function of the basalt fiber concentration of an applied coating. These coatings are acrylate based containing basalt fibers with $0.16 \mathrm{~mm}$ length with increasing basalt fiber concentration in the coatings 


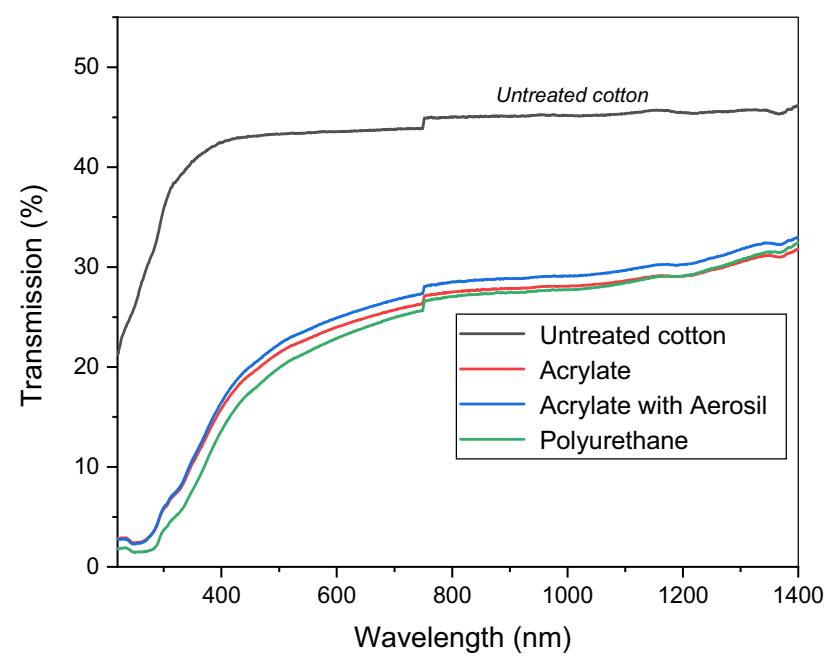

Fig. 13: Optical spectra of diffusive transmission. Compared are basalt fiber-containing coatings with different binder recipes. The used basalt fibers contain a length of $0.16 \mathrm{~mm}$ and are added to the coating recipe in a concentration of $20 \%$. The reflection spectrum of the untreated cotton fabric is shown as a reference

In Fig. 13, the transmission spectra of samples with the same concentration of basalt fibers $(20 \%)$ but with different binder recipes are compared. It is clearly seen that the type of used binder has nearly no influence on the optical transmission of the realized coated samples. It can be stated that the component determining the optical transmission of these coatings is the basalt fiber.

In contrast to UV-protective materials, IR-protective materials are based not only on the absorption of infrared-radiation. Also, the reflection of infraredradiation is an important mechanism here, which is especially used in metal coated textiles by the use of high reflective pigments. A good example in this field is the coating of cotton fabrics with thin metallic silver films. As a result, the nearly complete shielding of infrared radiation can be reached. ${ }^{39}$ Here the applied silver films increase the reflectivity for IR-light and thus, the transmission for this IR-light is decreased. ${ }^{39} \mathrm{~A}$ similar effect is reported for copper films on polyester and cotton. ${ }^{40}$ By application of $\mathrm{ZnO}$ nanoparticles, the IR-reflectance of black cotton fabrics can be increased. ${ }^{35}$ However, with other metal coatings, such as titanium, this effect cannot be reached. ${ }^{40}$

In contrast to these IR-reflecting materials, the developed basalt fiber coatings lead to a decrease in IR-reflection and simultaneously to a drastic reduction in the transmission for IR-light. The reason for this decrease is likely due to a strong absorption of IR-light by the basalt fibers. Because of the decreased transmission, the basalt fiber coated textiles can be used as protective materials against IR-light. The lower reflection of IR-light has the advantage that other materials or persons are not exposed by the reflected IR-light.

Altogether it can be stated that the developed basalt fiber coatings on textiles can lead to applications for simultaneous protection against UV-light and IR-light by only one coating application. Of course, the UVprotection in the spectral range of 370 to $400 \mathrm{~nm}$ could be further enhanced by addition of an organic UVabsorber especially dedicated for this spectral area of UV-light. However, the development of a combined application of basalt fibers and organic UV-absorbers in the same coating goes beyond the scope of the actual study.

Beside the changes of optical properties for UV-and IR-light, the optical properties for visible light and, by this, the coloration of samples is changed after the coating application. The reflection spectra exhibit a significant decrease in reflected light as a function of basalt fiber concentration added to the coating (Figs. 8 and 10). By this, a darker coloration after coating is determined. In addition to the optical spectroscopy, the sample coloration is determined by using CIE $L^{*} a^{*} b^{*}$ indices (Table 1). In comparison to the uncoated cotton reference, a clear change in CIE indices after coating application is determined. This change in CIE indices is increased as a function of basalt fiber concentration in the applied coating. The index for brightness $L^{*}$ is decreased from 85.33 as the value of the cotton reference down to 34.07 for the textile with the highest basalt fiber concentration. This result from CIE measurement is in good agreement with the reflection spectra determined by optical spectroscopy for the range of visible light (Fig. 8). Also, by the reflection measurements, a significant decrease in reflection is determined if basalt fibers are added in higher concentrations. In fact, basalt fibers exhibit a brown coloration, so a coating containing basalt fibers changes the color of the coated fabric to dark brown. The change of the index $a^{*}$ from negative to positive values after basalt fiber applications indicates a shift to a redder coloration of the samples after coating. The increase in the index $b^{*}$ after basalt fiber application indicates a change to a yellower coloration after coating. In fact, this is the red-brown coloration of basalt fiber which is transferred to the coated textile.

\section{Conclusions}

Short basalt fibers can be used as coating additives for polymer coatings applied onto textiles. The combination of basalt fibers with acrylate and polyurethane polymers is possible. According to the stability of the coating recipe, basalt fibers with fiber length of $1.6 \mathrm{~mm}$ or higher are less advantageous for use as a coating additive. Also, the use of these longer fibers leads to a negative impact on the textile comfort and the hand feeling of the finally gained textile. In contrast, by adding the shortest basalt fibers with length of $0.16 \mathrm{~mm}$, the mechanical properties of the textile are chained but the hand feeling of the coated fabric is still suitable. The surface topography of the realized coatings is determined by a parallel arrangement of 
Table 1: CIE $L^{*} a^{\star} b^{\star}$ indices of cotton samples with the acrylate-based coatings containing basalt fibers of 0.16 mm length with increasing basalt fiber concentration in the coating recipe

Samples, content of basalt fiber in coating recipe (\%)

\begin{tabular}{ccr} 
& Indices \\
\hline$L^{*}$ & $a^{*}$ & $b^{*}$ \\
\hline 81.36 & -0.30 & 3.84 \\
77.96 & -0.11 & 5.55 \\
74.73 & +0.07 & 6.91 \\
71.10 & +0.35 & 8.32 \\
64.38 & +0.77 & 10.52 \\
60.04 & +1.10 & 11.47 \\
41.34 & +2.31 & 13.75 \\
35.58 & +2.21 & 12.02 \\
34.07 & +2.07 & 11.29 \\
85.33 & -0.42 & 1.57 \\
\hline
\end{tabular}

some basalt fibers to each other, which occurs especially for medium to high concentration of applied basalt fibers in the coating recipe. The basalt fibercontaining coatings influence the optical properties of the coated substrate from the UV-range to near infrared light. The transmission of UV-light and IRlight can be decreased significantly, so an application in the area of UV-protection or IR-protection is possible. Nevertheless, it could be kept in mind for introduction of these coated materials for practical applications, and even for commercialization there is also the strong demand for the durability of the coated fabrics against influences like washing and rubbing. Washing and rubbing durability have to be at least sufficient. However, due to the limited size of this paper, durability aspect is not the scope and will be published separately.

Acknowledgments Open Access funding provided by Projekt DEAL. For helpful discussions and advice in the finishing laboratory, the authors would like to thank Mister Thomas Heistermann (Hochschule Niederrhein, Mönchengladbach, Germany). All product and company names mentioned in this article may be trademarks of their respected owners, even without labeling.

Open Access This article is licensed under a Creative Commons Attribution 4.0 International License, which permits use, sharing, adaptation, distribution and reproduction in any medium or format, as long as you give appropriate credit to the original author(s) and the source, provide a link to the Creative Commons licence, and indicate if changes were made. The images or other third party material in this article are included in the article's Creative Commons licence, unless indicated otherwise in a credit line to the material. If material is not included in the article's Creative Commons licence and your intended use is not permitted by statutory regulation or exceeds the permitted use, you will need to obtain permission directly from the copyright holder. To view a copy of this licence, visit http://creativecommons.org/licenses/by/4.0/.

\section{References}

1. Jamscheid, H, Mishra, R, "A Green Material from Rock: Basalt Fiber-A Review." J. Text. Inst., 107 923-937 (2016)

2. Singha, K, "A Short Review on Basalt Fiber." Int. J. Text. Sci., 1 19-28 (2012)

3. Militky, J, Kovacic, V, "Ultimate Mechanical Properties of Basalt Filaments." Text. Res. J., 66 225-229 (1996)

4. Mahltig, B, "Basalt Fibers.” In: Mahltig, B, Kyosev, Y (eds.) Inorganic and Composite Fibers, pp. 195-218. Woodhead Publishing, Duxford (2018)

5. Deak, T, Czigany, T, "Chemical Composition and Mechanical Properties of Basalt and Glass Fibers: A Comparison." Text. Res. J., 79 645-651 (2009)

6. Pico, D, Wilms, C, Seide, G, Gries, T, "Natural Volcanic Rock Fibers." Chem. Fibers Int., 61 90-92 (2011)

7. Overkamp, T, Mahltig, B, Kyosev, Y, "Strength of Basalt Fibers Influenced by Thermal and Chemical Treatments." $J$. Ind. Text., 47 815-833 (2018)

8. Gutnikov, SI, Zhukovskaya, ES, Popov, SS, Lazoryak, BI, "Correlation of the Chemical Composition, Structure and Mechanical Properties of Basalt Continuous Fibers." AIMS Mater. Sci., 6 806-820 (2019)

9. Dhand, V, Mittal, G, Rhee, KY, Park, S-J, Hui, D, "A Short Review on Basalt Fiber Reinforced Polymer Composites." Compos. B, 73 166-180 (2015)

10. Botev, M, Betchev, H, Bikiaris, D, Panayiotou, C, "Mechanincal Properties and Viscoelastic Behavior of Basalt FiberReinforced Polyproplyene." J. Appl. Polym. Sci., 74 523-531 (1999)

11. Czigany, T, Vad, J, Poloskei, K, "Basalt Fiber as a Reinforcement of Polymer Composites." Period. Polytech. Ser. Mech. Eng., 49 3-14 (2005)

12. Mingchao, W, Zuoguang, Z, Yubin, L, Min, L, Zhijie, S, "Chemical Durability and Mechanical Properties of Alkali- 
Proof Basalt Fiber and Its Reinforced Epoxy Composites." J. Reinf. Plast. Compos., 27 393-407 (2008)

13. Sim, J, Park, C, Moon, DY, "Characteristics of Basalt Fiber as a Strengthening Material for Concrete Structures." Compos. B, 36 504-512 (2005)

14. Afroz, M, Patnaikuni, I, Venkatesan, S, “Chemical Durability and Performance of Modified Fiber in Concrete Medium." Constr. Build. Mater., 154 191-203 (2017)

15. Quagliarini, E, Monni, F, Bondioli, F, Lenci, S, "Basalt Fiber Ropes and Rods: Durability Tests for Their Use in Building Engineering." J. Build. Eng., 5 142-150 (2016)

16. Hong, H, Qing, H, "Knitting of Basalt Filament Yarn." Text. Res. J., 81 690-697 (2010)

17. Bednar, M, Hajek, M, "Hitzeschutztextilien aus neuartigen Basalt-Filamentgarnen." Techn. Textilien, 43 252-254 (2000)

18. Licai, H, Weidong, Y, "Comparison of Thermal Protective Performance of Aluminized Fabrics of Basalt Fiber and Glass Fiber." Fire Mater., 35 553-560 (2010)

19. Gilewicz, P, Frydrych, I, Cichocka, A, Pinkos, J, Hrynyk, R, Orawiec, G, Zielinski, J, "Measurement of Joint Durability Between Aluminum Foil and Basalt Fabrics." Fibres Text. in East. Eur., 27 81-84 (2019)

20. Ali, AHM, Yu, W, "Thermal Protective Performance of Multilayer Fire Fighting Fabric.” Int. J. Cloth. Sci. Technol., 26 235-246 (2014)

21. Hao, LC, Yu, WD, "Evaluation of Thermal Protective Performance of Basalt Fiber Nonwoven Fabrics." J. Therm. Anal. Calorim., 100 551-555 (2010)

22. Kasparova, M, Wiener, J, Shahidi, S, "Multilayer Assembly for Protection Against Laser Light." J. Text. Inst., 111 226234 (2020)

23. Yasir, M, Amir, N, Ahmand, F, Ullah, S, Jimenez, M, "Effect of Basalt Fibers Dispersion on Steel Fire Protection Performance of Epoxy-Based Intumescent Coatings." Prog. Org. Coat., 122 229-238 (2018)

24. Hao, Y, Liu, F, Shi, H, Han, E, Wang, Z, "The Influence of Ultra-Fine Glass Fibers on the Mechanical and Anticorrosion Properties of Epoxy Coatings." Prog. Org. Coat., 71 188-197 (2011)

25. Bilisik, K, Yolacan, G, “Abrasion Properties of Upholstery Flocked Fabrics." Text. Res. J., 79 1625-1632 (2009)

26. Bilisik, K, Demiryurek, O, Turhan, Y, "Mechanical Characterization of Flocked Fabric for Automobile Seat Cover." Fibers Polym., 12 111-120 (2011)

27. Mahltig, B, Zhang, J, Wu, L, Darko, D, Wendt, M, Lempa, E, Rabe, M, Haase, H, "Effect Pigments for Textile Coating: A Review of the Broad Range of Advantageous Functionalization." J. Coat. Technol. Res., 14 35-55 (2017)
28. Saravanan, D, "UV Protection Textile Materials." AUTEX Res. J., 7 53-62 (2007)

29. Mahltig, B, Haufe, H, Böttcher, H, "Functionalisation of Textiles by Inorganic Sol-Gel Coatings.” J. Mater. Chem., 15 4385-4398 (2005)

30. Rubeziene, V, Minkuviene, G, Baltusnikaite, J, Padleckiene, I, "Development of Visible and Near Infrared Camouflage Textile Materials." Mater. Sci. (Medziagotyra), 15 173-177 (2009)

31. Colliex, C, Kohl, H, Elektronenmikroskopie. Wissenschaftliche Verlagsgesellschaft mbH, Stuttgart (2008)

32. Brennan, P, Fedor, C, "Sunlight, UV \& Accelerated Weathering." Paint Resin, 5817 (1988)

33. Mahltig, B, Böttcher, H, Rauch, K, Dieckmann, U, Nitsche, R, Fritz, T, "Optimized UV Protecting Coatings by Combination of Organic and Inorganic UV Absorbers." Thin Solid Films, 485 108-114 (2004)

34. Mahltig, B, Textor, T, Nanosols and Textiles. World Scientific Publishing, Singapore (2008)

35. Soumya, S, Nishanth Kumar, S, Peer Mohmed, A, Ananthakumar, S, "Silanated Nano ZnO Hybrid Embedded PMMA Polymer Coatings on Cotton Fabrics for Near-IR Reflective, Antifungal Cool-Textiles." New J. Chem., 40 7210-7221 (2016)

36. Becheri, A, Dürr, M, Lo Nostro, P, Bagliono, P, "Synthesis and Characterization of Zinc Oxide Nanoparticles: Application to Textiles as UV-Absorbers." J. Nanopart. Res., 10 679-689 (2008)

37. Paul, R, Bautista, L, De la Varga, M, Casals, E, Puntes, V, Marsal, F, "Nano-Cotton Fabrics with High Ultraviolet Protection." Text. Res. J., 80 454-462 (2010)

38. Hoque, MT, Mahltig, B, "Realisation of Polyester Fabrics with Low Transmission for Ultraviolet Light." Color. Technol., 136 346-355 (2020). https://doi.org/10.1111/cote.12470

39. Jiang, S, Miao, D, Xu, J, Shang, S, Ning, X, Zhu, P, "Preparation and Characterization of Shielding Textiles to Prevent Infrared Penetration with Ag Thin Films." J. Mater. Sci. Mater. Electron., 28 3542-3547 (2017)

40. Miao, D, Jiang, S, Liu, J, Ning, X, Shang, S, Xu, J, "Fabrication of Copper and Titanium Coated Textiles for Sunlight Management." J. Mater. Sci. Mater. Electron., 28 9852-9858 (2017)

Publisher's Note Springer Nature remains neutral with regard to jurisdictional claims in published maps and institutional affiliations. 